\title{
THE DESIGN AND DEVELOPMENT OF DATA HIDING USING DEEP LEARNING
}

\section{${ }^{1}$ K.RAGHAVENDRA PRASAD ${ }^{2}$ Dr NEERAJ SHARMA}

${ }^{1}$ Research Scholar, BE, M Tech, (PhD in COMPUTER SCIENCE AND ENGINEERING ), Sri Satya Sai University of Technology \& Medical Sciences, Sehore,Madhya Pradesh, India

${ }^{2}$ Associate Professor, CSE Department Sri Satya Sai University of Technology \& Medical Sciences,Sehore, Madhya Pradesh, India

Abstract : Data hiding is one of the most prominent and important topics. The importance of protecting sensitive data has always been recognized from time immemorial. Steganography is the craft of secured or shrouded composing; the term itself goes back to the fifteenth century, when messages were physically covered up. In present day steganography, the objective is to clandestinely impart an advanced message. The steganographic procedure puts a shrouded message in a vehicle medium, called the transporter. The bearer might be openly noticeable. For included security, the shrouded message can likewise be encoded, accordingly expanding the apparent haphazardness and diminishing the probability of substance disclosure regardless of whether the presence of the message identified. Great acquaintances with steganography and steganalysis.

Index Terms -Information Hiding, Deep Learning, Natural language processing (NLP), Convolutional Neural Networks (CNN)

\section{INTRODUCTION}

In information hiding, secret information is hidden by modifying the pixels of a cover image. Detectors can detect the hidden information if the cover image is modified. In 2014, experts proposed coverless information hiding as a way to prevent this problem completely and resist steganalysis fundamentally. By coverless information hiding, the secret information is used as a driver to find or generate a stego image corresponding to the secret information. It is not necessary to change the cover image during this process. To create a mapping relationship between secret information and feature information, a mapping dictionary is constructed. A mapping dictionary is shared between the sender and receiver. The transmitter transmits an image or text that maps to the secret information and the receiver extracts the secret information based on the mapping relationship, thus resisting steganalysis. It is widely recognized that coverless information hiding can be divided into two 
branches.into two branches. There is one type of coverless text information hiding on the transits of text. Natural language processing (NLP) has gained prominence, as demonstrated by the effectiveness of algorithms such as word2vec and LSTM . There are some achievements that combine the advanced knowledge of NLP with the coverless text information hiding technique, due to the strong similarity between the mapping relationship of word embedding and text coverless information hiding. Alternatively, one can hide the coverless information in images, known as image transits .

Data security is a testing issue with the high development rate of internet. Cryptography, Steganography and Digital watermarking strategies are widely used for data security for various purposes. Cryptography is utilized mainlyfor secure correspondence with the presence of scrambled message. Steganography is likewise utilized for secure correspondence however presence of the message is hidden. Digital watermarking is a particular sort of steganography in which we shroud the data to assert the responsibility for media, to control the duplicate of computerized media and the unlawful conveyance of interactive media information. Presently more difficulties are observed in distributionof illicit duplicate of visuals and audio information, with copyright encroachment and unlawful possession. Ordinarily interactive media information i.e. images, sound, video and so on are wrongfully flowed, transmitted and abusing licensed innovation rights and thereby causing misfortunes and great losses to the proprietor of information.

2. LITERATURE SURVEY :G.E. Hinton et al. [Hinton and Salakhutdinov (2006)] proposed the technique for unaided pre-preparing to streamline the underlying estimation of system loads, and afterward fine-tune the loads, which opened the prelude of profound learning.

Profound learning is basically isolated into three kinds: Supervised learning, unaided learning and support learning. Directed learning alludes to AI with both trademark worth and name esteems in info information. By figuring the blunder between the system yield worth and mark esteem, it is relied upon to prepare the system iteratively to locate the best yield esteem. The issues that should be explained in managed learning can be isolated into two classifications: relapse [Fu, Gong, Wang et al. (2018)] and characterization [Gurusamy and Subramaniam (2017)]. As a basic grouping task, picture arrangement is an examination field that draws in much consideration. The order of 1,000 classifications on Image Net [Russakovsky, Deng, Su et al. (2014)] added to the advancement of CNN, for example, VGG. 
As of now, some prominent managed learning calculations are spoken to by convolutional neural system $(\mathrm{CNN})$ and profound conviction organize $(\mathrm{DBN})$. Extraordinary learning machine [Gautam, Tiwari and Leng (2017)] is an AI dependent on feed forward neuron organize. It is additionally a sort of directed learning. It is utilized for forecast [Dutta, Murthy, Kim et al. (2017)], order, etc. The objective of solo learning is to locate some normal highlights, structures, or relationships between's the trademark estimation of information through AI. Unaided learning techniques, for example, autoencoder [Kingma and Welling (2013)], profound boltzmann machine.

The WGAN (Wasserstein GAN) proposed by Arjovsky et al. in 2017 viably improved GAN [Arjovsky, Chintala and Bottou (2017)]. It takes care of the issue of shaky GAN preparing, proposes powerful techniques to guarantee the decent variety of produced tests, utilizes explicit cross-entropy capacity to show the preparation procedure, and utilizations multi-layer neural system to finish preparing without planning a particular system structure. Least squares GAN (LSGAN) [Mao, Li, Xie et al. (2017)] advances GAN by utilizing a smoother and non-immersing slope misfortune work in the discriminator.

\section{A NEURAL NETWORK - Convolutional} Neural Networks have appeared to learn structures that relate to sensible highlights. These highlights increment their degree of reflection as we go further into the system. Utilizing a ConvNet will take care of the considerable number of issues referenced previously. Right off the bat, the convnet will have a smart thought about the examples of characteristic pictures, and will have the option to settle on choices on which zones are repetitive, and more pixels can be covered up there. By sparing space on excess regions, the measure of shrouded data can be expanded. Since the engineering and the loads can be randomized, the precise manner by which the system will shroud the data can't be known to anyone who doesnt have the loads.

\section{THE ARCHITECTURE}

The whole system design is shockingly like Auto Encoders. All in all, auto-encoders are made to replicate the contribution after a progression of changes. By doing this, they find out about the highlights of the information dissemination.

For this situation, the design is somewhat unique. Rather than simply repeating pictures, the engineering needs to conceal a picture, just as duplicate an other picture. 


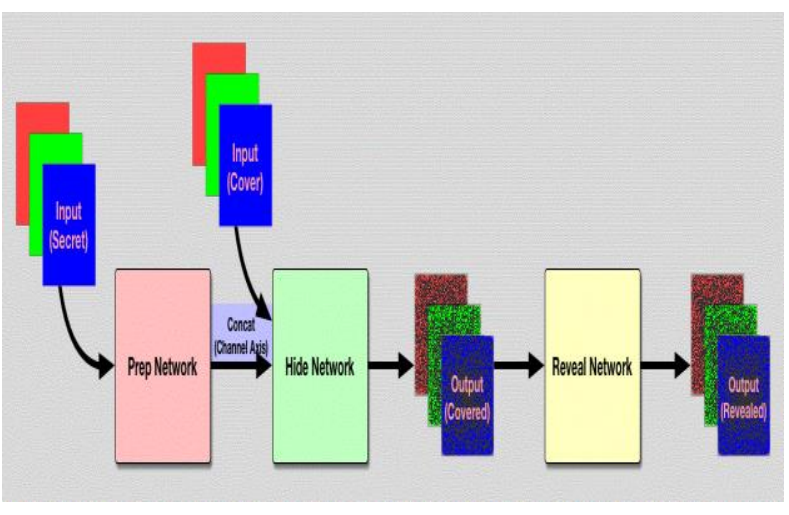

Figure1.1 : Network Architecture

The entire structure comprises of 3 Parts: The Prepare Network, The Hide Network, and The Reveal Network.

The Prep Network takes in the mystery picture, and 'sets it up. The Hide Network takes in the Output of the Prep organize just as the Cover Image. These two sources of info are first linked over the Channels Axis. The Hide Network yields a picture, which is the Hidden Image. This is the Image that contains the Secret, however resembles the Cover.

So as to recover the Secret Image, it should be passed to a Reveal Network. The Reveal Network will yield an Image, which resembles the Secret.

The genuine design of every one of the systems is generally comparable, and there is a great deal of space for experimentation. I utilized $4(3 \times 3),(4 \times 4) \&$ (5x5) piece convolutions on the input(50 maps), before concating. At that point I did another 3 convolutions on the connected component maps. After that, I did a $1 \mathrm{x} 1$ convolution to create 3 channels. You can find out about the genuine subtleties in the execution code, and the graph in my repo.

\section{The Network Losses}

The Loss is genuinely clear. It is:

$$
\mathcal{L}\left(c, c^{\prime}, s, s^{\prime}\right)=\left\|c-c^{\prime}\right\|+\beta\left\|s-s^{\prime}\right\|
$$

Where $\mathrm{c}$ is the info spread, $\mathrm{c}^{\prime}$ is the secured picture. s and s' are the mystery info, and mystery spread pictures, separately.

The misfortune is the standard MSE between the genuine spread picture and the created secured picture , and $\beta^{*}(\mathrm{MSE}$ between real mystery picture and the delivered uncovered picture). Beta is a hyper parameter that controls the amount of the mystery ought to be remade. In this manner the misfortune improves for the accompanying explanation.

"The secured picture should look near the spread picture, and when uncovered, the uncovered picture should look extremely near the mystery picture".

Since the capacity is differentiable, the whole system can be prepared start to finish.

The paper reports results that are generously superior to existing techniques. There is an apparatus called Steg Expose, which can discover whether a picture has something covered up. It is genuinely simple to see whether the picture is altered on the off chance that it is concealed 
utilizing existing strategies. Be that as it may, this technique can trick Steg Expose.

\section{IMAGE WATERMARKING USING QR DECOMPOSITION AND LTSVR IN WAVELET DOMAIN}

The two areas of advanced picture watermarking are spatial and frequency domain. It has been shown that frequency domain watermarking is more resistant to attacks than spatial space watermarking. The experts whose work includes gentic algorithm calculations and their blend based half and half picture watermarking framework are planning to expand the subtlety and robustness of their generative algorithm calculations. The incapability to measure impalpability and power against picture handling assaults has been achieved due to the versatile learning capacity of picture informational indexes and the strong speculation capacity of these AI calculations. Here, a recently proposed LTSVR AI approach is applied to picture watermarking. We have assessed the hypothesis execution of LTSVR on available datasets that are acquired from the UCI storage facility and against uproarious datasets. The work presented in this paper examines the adaptive learning capacity of LTSVR onto picture watermarking criticalities and its ability to cope with disorderly datasets.

\subsection{Watermark Insertion Algorithm}

Consider a gray scale image

$\operatorname{Img}=\{\operatorname{Img}(r, s): 1 \leq r \leq M 1,1 \leq s \leq M 2\}$ of order ofM1 $\times M 2$. In this work a binary watermark logo of order of $N 1 \times N 2$ is used for embedding and extracting purpose. The approach for inserting the watermark into the host image is as:

Firstly the scrambled image $S_{\mathrm{m}}$ of the original binary watermark is formed using Arnold transformation [Wu et al., 2009]. Then it transformed into 1-Dvector to insert into the host. That is $S W_{m}=\left\{w_{k}: k=\right.$ $\left.1,2, \ldots l_{w}\right\}$ where $l_{w}$ is length of watermark $w_{k}=$ $\{0,1\}$.

And using one level LWT, the host image is divided into the low frequency sub-band and detailed sub bands denoted by LL and LH, HL, HH respectivelywith order $M_{L} \times N_{L}$ where. $M_{L}=\frac{M 1}{2^{r}}$ ， $N_{L}=\frac{M 2}{2^{r}}$ Here decomposition level is denoted by $\mathrm{r}$. The lifting coefficients of low frequency sub band are dividedinto blocks of order of 44 . Fuzzy entropy [Kumar et al., 2011] of every block is calculated and arranged in descending order.

Perform QR decomposition to the selected blocks of low frequency sub band using Eq. (4.1) to get the $Q$ and R matrix of order equal to the block size. From the experimental results, it is found that $r_{1 ; 4}$ is the 
appropriate element to embed the scrambled watermark. The feature vector formedusing the upper triangularelements

$\left\{r_{1,1}, r_{1,2} r_{1,3}, r_{2,2} r_{2,3}, r_{2,4}, r_{3,3}, r_{3,4}, r_{4,4}\right\}$ are supplied as input to LTSVR corresponding to target vector made up ofthe element $r_{1,4}$.Thus an image dataset is constructed using the featuresvectors of all the selected non overlapping blocks of order of $\mathrm{m} 1$. (Here $1=10)$

Based upon fuzzy entropy, the dataset constructed using the suitable features of the image blocks for training of the LTSVR. That is

$$
D S=\left\{\begin{array}{l}
\left(x_{i}, d_{i}\right) \in R^{9} \times R: i=1,2, \ldots, m \\
=\left\{\left(r_{1,1}, r_{1,2}, r_{1,3,}, r_{2,2,}, r_{2,3}, r_{2,4}, r_{3,3,}, r_{3,4}, r_{4,4}\right), r_{1,4}\right\}
\end{array}\right\}
$$

Where the target output vector consists of $r_{1 ; 4}$ element of each selected blockand remaining nine upper triangular elements of each block is supplied as input to LTSVR. The feature vector of odd number of selected regions are used to train the LTSVR that is . $\mathrm{DS}=\left\{\left(x_{i} \mid d_{i}\right): i=1,3,5 \ldots . m\right\} . \quad$ The function obtained after the training of LTSVR using Eq. (3.11) is used to nd the predicted value corresponding to the target vector of even number of blocks. On comparing the predicted value corresponding to the target vector $d_{i}=\left\{\left(r_{1,4}: i=2,4,6, \ldots, m\right)\right\}$, the watermark bits are inserted as:

$$
r_{1,4}^{\prime}= \begin{cases}\max \left(r_{1,4}, r_{1,4}^{\text {LTSVR }}+\alpha\right) & \text { if } w m_{-} \text {bit }=1 \\ \min \left(r_{1,4}, r_{1,4}^{\text {LTSVR }}-\alpha\right) & \text { otherwise }\end{cases}
$$

where, $r_{1,4}^{\prime}$ is the watermark embedded value after inserting the watermarkwhich is replaced by the $r_{1,4}$ of $\mathrm{R}$ of the selected region $r_{1,4}^{L T S V R}$ is the predicted value found by the training function of LTSVR, denotes the strength of watermark and wm bit represents bit of scrambled image. After performing a number of experiments, the value of $=20$ is chosen to minimize the trade o between two conflicting requirements.

\section{PERFORMANCE EVALUATION}

The presentation of the proposed approach is assessed utilizing the subtlety of the watermark and its vigour The quality boundary PSNR and BER relating to the watermarked picture and removed watermark alongside the representation of images are demonstrated in Fig. 4.4 when no picture handling assault is performed. The Robustness Comparison on Boat picture with [Song et al. 2011] 


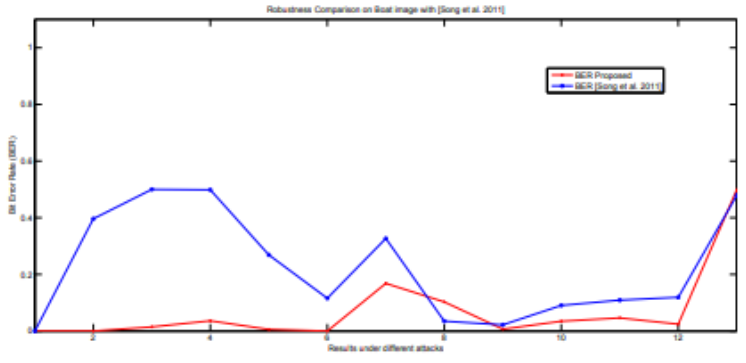

Fig. 1.2 BER value comparisons against image processing operations on Lena image with [Song et al., 2011]

PSNR esteem more than 40dB as demonstrated in Fig. 4.4 of the watermarked rendition pictures shows the great nature of watermarked pictures with high indistinctness of the watermark. The precise watermark extraction utilizing proposed approach when no assault is performed is confirmed by the zero BER esteem and appeared in Fig. 4.4. The heartiness of the methodology depicted in this paper is researched by per-framing a few sorts of pictures preparing tasks, for example, obscuring, salt and pepper clamour, Gaussian commotion, histogram levelling, JPEG pressure, gamma adjustment, middle separating, normal sifting, scaling and editing and so forth on all the watermarked pictures followed by watermark extraction measure. The visual nature of recuperated watermark as estimated by the BER esteem against all the assaults is appeared in Table 1.1 relating to all watermarked pictures. BER esteems appeared in Table 1.1, it is seen that comparing to all the assaults the proposed approach has lower BER esteem which means that the removed watermark has great visual quality and conspicuous.

Comparison Results: The viability of the plan introduced in this paper is inspected by contrasting the strength against assaults and the technique portrayed by [Song et al., 2011] 102

Table 1.1 Visual Quality of Extracted Watermark along with Corresponding BER value against Image Processing attacks on Lena and Elaine images

\begin{tabular}{|c|c|c|c|c|}
\hline Attacks $\downarrow$ & BER $($ Lena $)$ & $\begin{array}{c}\text { Extracted } \\
\text { Water- } \\
\text { mark (Lena) }\end{array}$ & BER(Elaine) & $\begin{array}{c}\text { Extracted } \\
\text { Water- } \\
\text { mark (Elaine }\end{array}$ \\
\hline Gaussian Blurring & 0.0127 & & 0.0088 & \\
\hline Salt \& Pepper noise $(0.02)$ & 0.1028 & & 0.1562 & \\
\hline Gaussian noise $(0.10)$ & 0.0345 & & 0.0453 & \\
\hline Gaussian noise $(0.20)$ & 0.1683 & & 0.1763 & \\
\hline Histogram Equalization & 0.0068 & & 0.0088 & \\
\hline JPEG $(Q F=80)$ & 0 & & 0 & \\
\hline JPEG $(Q F=60)$ & o & & 0.0029 & \\
\hline Gamma Correction & 0.0098 & & 0.0088 & \\
\hline Sharpening & 0.0068 & & 0.0186 & \\
\hline Scaling & 0.0059 & & 0.0039 & \\
\hline Average Filtering & 0.0361 & & 0.0322 & \\
\hline Median Filtering & 0.0146 & & 0.0342 & \\
\hline Cropping (25\%) & 0.0244 & & 0.0244 & \\
\hline
\end{tabular}

on Lena picture. For reasonable correlation, same sorts of assaults are executed on Lena picture and afterward watermark extraction methodology is performed. The results of the watermark extraction methodology estimated by the BER esteem against assaults are given in Table 1.1 and appeared in Fig. 1.2. 
In this work, a powerful methodology of dark scale picture watermarking utilizing LTSVR and through the blend of wavelet change and QR decay is depicted for duplicate right insurance applications. Fluffy entropy isn't simply used to dispose of the districts of the picture which are not pertinent to implant the watermark but rather likewise diminishes the time multifaceted nature. Determination of LL sub band utilizing LWT and proper coefficient choice of every district utilizing $\mathrm{QR}$ disintegration brings about upgrading the exhibition as estimated by impalpability and heartiness. The power estimated by various types of assaults performed on test pictures is refined by the great speculation property of LTSVR as uncovered from the test results utilizing proposed approach. The mixed watermark acquired utilizing Arnold change gives the security to the first watermark. The exploratory and correlation results on di erent finished pictures with the current techniques demonstrate that the methodology depicted in this paper achieves subtlety just as strength.

\section{CONCLUSION}

A computerized watermark is a method that is incredibly commonly used for giving copyright protection, duplicate assurance, and ownership attestation of advanced visual and audio content. In computerized watermarking, the copyright data is inserted straightforwardly into the advanced substance so that it generally endures even in the wake of handling assaults. The commitment of this proposition is in the territory of computerized picture watermarking by utilizing canny AI calculations to set exchange $\mathrm{o} \quad \mathrm{s}$ between different watermarking boundaries. AI methods are applied in various watermarking schemes to accomplish streamlining and to get better outcomes as analyzed than contemporary procedures. We have effectively set exchange o s between watermark strength and impalpability, while keeping the payload steady. The examination result of this theory is in three-overlay. The target of our examination is to create hearty and vague picture water-checking plan for copyright security, duplicate insurance and proprietorship affirmation like applications utilizing AI calculations. We have created calculations which full our targets. We have covered numerous issues of picture watermarking yet a few issues are there for future examination recorded underneath.Our proposed plans are profoundly hearty against various picture handling assaults yet it isn't appropriate for revolution and interpretation.

\section{REFERENCES}

1. Ying Zoua , Ge Zhang b,c, Leian Liua 2019 "Research on Image Steganography Analysis Based on Deep Learning" College of Information Science and Technology, Zhongkai University of Agriculture and 
Engineering, No. 501, Zhongkai Rd, Haizhu District, Guangzhou, China.

2. Jiren Zhu 2018 "HiDDeN: Hiding Data With Deep Networks“ Computer Science Department, Stanford University.

3. Gautan, Aruna Tiwari, Qian leng "On The Construction of Extreme Learning Machine for Online and Offline One-Class Classification An Expanded Toolbox" January 2017 Neurocomputing 261

DOI: 10.1016/j.neucom.2016.04.070 .

4. Diederik P. Kingma, Max Welling, Machine Learning Group Universiteit van Amsterdam "Auto-Encoding Variational Bayes", arXiv:1312.6114v10 [stat.ML] 1 May 2014.

5. Grégoire Montavon Klaus-Robert Müller, Deep Boltzmann Machines and the Centering Trick, DOI : $\quad$ https://doi.org/10.1007/978-3-64235289-8_33.

6. Ian J. Goodfellow, Jean Pouget-Abadie, Mehdi Mirza, Bing Xu, David Warde-Farley, Sherjil Ozair, Aaron Courville, Yoshua Bengio, Generative Adversarial Networks(GAN), ArXiv Oct-25 to 31, Submitted on 10 Jun 2014.


arXivpreprint arXiv, 2015 Massively parallel methods for deep reinforcement Learning,

8. Christoph FeichtenhoferHaoqi FanJitendra MalikKaiming he "SlowFast Networks for Video
Recognition” October 2019 DOI: 10.1109/ICCV.2019.00630 Conference: 2019 IEEE/CVF International Conference on Computer Vision (ICCV)

9. Holub, V.; Fridrich, J.; Denemark, T. (2014): Universal distortion function for steganography in an arbitrary domain. EURASIP Journal on Information Security, vol. 2014, no. 1, pp. 1.

10. Holub, V.; Fridrich, J. (2012): Designing steganographic distortion using directional filters. IEEE International Workshop on Information Forensics and Security, vol. 2, no. 4, pp. 234-239.

11. evný, T.; Filler, T.; Bas, P. (2010): Using highdimensional image models to perform highly undetectable steganography. Lecture Notes in Computer Science, vol. 6387, pp. A Survey of Image Information Hiding Algorithms 451 161177.

12. Fridrich, J. (1999): Protection of digital images using self-embedding. Symposium on Content Security and Data Hiding in Digital Media, New Jersey Institute of Technology.

13. He, H. J.; Zhang, J. S.; Tai, H. M. (2009): Selfrecovery fragile watermarking using blockneighborhood tampering characterization. International Workshop on Information Hiding, pp. 132-145. 
14. He, H.; Chen, F.; Tai, H.; M.; Kalker, T.; Zhang, J. (2012): Performance analysis of a block-neighborhood-based self-recovery fragile watermarking scheme. IEEE Transactions on Information Forensics and Security, vol. 7, no. 1, pp. 185-196.
15. Dorairangaswamy, M. A. (2009): A novel invisible and blind watermarking scheme for copyright protection of digital images. International Journal of Computer Science and Network Security, vol. 9, no. 4, pp. 71-78. 